\title{
Effect of Silver Diamine Fluoride and Proanthocyanidin on Mechanical Properties of Caries-Affected Dentin
}

\author{
Maryam Firouzmandi ${ }^{1}$ Fereshteh Shafiei ${ }^{1} \quad$ Zahra Jowkar $^{1} \quad$ Fereshteh Nazemi $^{2}$ \\ 1Oral and Dental Disease Research Center, Department of Operative \\ Dentistry, School of Dentistry, Shiraz University of Medical \\ Sciences, Shiraz, Iran \\ ${ }^{2}$ Student Research Committee, School of Dentistry, \\ Address for correspondence Zahra Jowkar, DMD, MsD, \\ School of dentistry, Ghasredasht Ave, Shiraz, Iran \\ (e-mail: zahrajowkar66@gmail.com).
}

Shiraz University of Medical Science, Shiraz, Iran

Eur J Dent 2019;13:255-260

\begin{abstract}
Keywords

- silver diamine fluoride

- grape seed extract

- Knoop hardness

- modulus of elasticity

- caries-affected dentin
\end{abstract}

Objectives Inner carious dentin is specified with decreased minerals and collagen cross-links but without protein denaturation. Current minimally invasive dentistry concepts recommend removal of only the outer layer of carious dentin and biomodification of repairable inner carious dentin. The present study aims to investigate the possibility of functional repair of this layer using silver diamine fluoride (SDF) and grape seed extract (GSE).

Materials and Methods Molar teeth with occlusal caries were used to prepare caries-affected dentin specimens for hardness and elastic modulus measurements. The specimens of each test were divided randomly into four equal groups. In the GSE group, the specimens were immersed in 6.5\% GSE solution for 10 minutes. In the SDF group, the specimens underwent a topical application of a 30\% SDF. In the GSE+SDF group, first the specimens were immersed in GSE and then exposed to SDF. In the SDF+GSE group, first SDF was applied and then the specimens were immersed in GSE. Microhardness measurements were taken at baseline and after treatment. A control group with distilled water treatment was also prepared for elastic modulus measurements.

Statistical Analysis One-way analysis of variance and post-hoc tests were used for statistical analysis.

Results There were significant differences in $\mathrm{H}_{1}-\mathrm{H}_{0}$ (final hardness-baseline hardness) among the groups. Baseline and final hardness of each group was also significantly different (SDF>SDF+GSE>GSE>GSE+SDF). Elastic modulus of SDF and SDF+GSE increased compared to the control group.

Conclusions SDF and SDF+GSE treatment can be recommended to increase hardness and elastic modulus of caries-affected dentin.

\section{Introduction}

Dentin caries initiates with dissolution of its inorganic component, hydroxyapatite crystals, by acids, mainly lactic acid. Acids are metabolic by-products of cariogenic bacteria. ${ }^{1}$ As the carious lesion progresses, the organic matrix of dentin is invaded by bacterial toxins and some intrinsic proteases known as matrix metalloproteases (MMPs)., ${ }^{2,3}$ These changes cause a reduction in microhardness of dentin. Elastic modulus and intrinsic strength of dentin matrix are also reduced. ${ }^{4}$ The resultant lesion consists of different zones with differences in their mineral content, collagen destruction, bacterial invasion, and mechanical properties. $^{5-8}$ 
Contemporary minimally invasive dentistry approach is increasingly oriented toward removal of only the outer layer of carious dentin, which is infected and considered to be non-remineralizable. This concept emphasizes arresting and remineralizing the inner layer of carious dentin with either solution chemistry or more fundamental tissue engineering approaches. ${ }^{9}$ Caries-affected dentin is demineralized and contains a few bacteria. It has been demonstrated that the ultimate tensile strength of the matrix of caries-affected dentin is lower than that of normal dentin. This can be related to the hydrolytic effect of bacterial or host enzymes on collagen matrix. ${ }^{10}$ There is evidence indicating that the condition of collagen matrix can influence mineral deposition and dispersion within dentin. ${ }^{11,12}$ Hence, dentin biomodification approaches should be targeted at residual bacteria, repair of collagen matrix, and replacement of lost minerals.

Modification of collagen matrix by potential therapeutic agents should be considered to enhance the stability of collagen matrix. ${ }^{13}$ Extrinsic collagen cross-linking agents may increase the mechanical stability of collagen matrix and its resistance to caries progression. ${ }^{14,15}$ Another approach to stabilize collagen matrix is inhibition of the proteolytic activity of MMPs in carious dentin. ${ }^{16,17}$

Proanthocyanidin (PA) in grape seed extract (GSE) is a natural collagen cross-linker that can improve the mechanical properties of collagen ${ }^{15}$ and reduce its susceptibility to enzymatic degradation. ${ }^{18}$ This potential therapeutic agent might help in functional repair of pathological dentin lesions.

Silver diamine fluoride (SDF) has been shown to remineralize caries, reduce caries formation, and prevent Streptococcus mutans biofilm growth on demineralized dentin specimens. ${ }^{19,20}$ There is evidence indicating that SDF can inhibit collagen degradation ${ }^{21}$ and MMPs activity. ${ }^{22}$ These capacities could be the reason for its success in arresting caries in clinical trials.

Biomodification of carious dentin intends to re-establish the functionality of the affected tissue. The new tissue restoration approaches, rather than traditional dig and fill procedures, bring forth a need to understand structure/properties relationship from a biomechanical perspective. ${ }^{9}$ The assessment of mechanical changes is often used to evaluate caries severity. ${ }^{6}$ Hence, a more appropriate end point to evaluate attempts at dentin repair should be the establishment of mechanical properties consistent with the normal tissue.

Dentin is the major structural constituent of human tooth; therefore, its mechanical properties are major determinants of tooth response to mechanical loading. It has been reported that the underlying soft dentin might compromise the fracture strength of a composite resin filling. ${ }^{23}$ Bertassoni et al evaluated the methods used to assess the efficacy of dentin remineralization. Among these methods, mechanical tests provide useful information about the recovery of dentin. However, it has been proposed that available literature does not supply adequate data on this issue. ${ }^{11}$

Microhardness measurements have been broadly used to evaluate biomechanical properties of dentin. Elastic modulus is one of the most important properties of dentin with regard to tooth strength. It is influenced by complex interactions between the ingredients and the microstructure. ${ }^{11}$ The present study aimed to investigate whether a synergistic effect exists between SDF and GSE to increase microhardness and elastic modulus of caries-affected dentin. The null hypothesis ran as follows: SDF, GSE, and the combination of these two agents do not influence hardness and elastic modulus of caries-affected dentin.

\section{Materials and Methods}

\section{Specimen Preparation}

Ninety extracted human molars, with occlusal caries radiographically, extending into the middle third of dentin with no prior restorations, were collected. ${ }^{24}$ The patients' informed consent was obtained under a protocol reviewed and approved by the Ethics Committee of the university. The teeth were immediately placed in a solution of $0.5 \%$ chloramine-T and used within 1 month of extraction.

Sixty teeth were used in the hardness test. To prepare the specimens for the hardness test, the roots were cut and the specimens were embedded in self-curing acrylic cylinders. Occlusal enamel was removed using a water-cooled saw (Isomet Low Speed Saw; Buehler Ltd., Lake Bluff, Illinois, United States). The entire cut surface was flooded with cariesdetecting solution to stain the lesion (Kuraray Medical Inc., Tokyo, Japan). The outer layer of the lesion that was stained in red was removed with a round carbide bur to reach the light pink zone. ${ }^{25}$ The excavated area was visually inspected using the North Carolina Dentin Sclerosis Scale, which yielded category 4 . In this category, dentin is glassy in appearance, dark yellow or slightly brownish, with the majority of the dentin exhibiting transparency. ${ }^{4,10}$ The surface of dentin was flattened using 600 to 1200-grit silicon carbide papers and polished with 1 and 1/4 $\mu \mathrm{m}$-grit diamond paste (FGM Dental Products, SP, Brazil). A layer of nail varnish was applied on the surface of the specimens except on the caries-affected dentin to define the test area.

Thirty teeth were used to determine elastic modulus. In order to prepare the specimens for tensile test, cariesaffected dentin area was determined as described in previous paragraph. Then multiple mesiodistal vertical cuts were made through the center of the lesion using a water-cooled saw to prepare dentin slabs with $0.5 \pm 0.1$ thickness. Two slabs from each tooth were selected and prepared to hourglass-shaped specimens using an ultrafine diamond bur mounted in a water-cooled high-speed handpiece. The test site was $0.5 \pm 0.1 \mathrm{~mm}$ in width and $0.5 \pm 0.1 \mathrm{~mm}$ in thickness, with a cross-sectional area of approximately $0.25 \mathrm{~mm}^{2}{ }^{10}$

\section{Experimental Treatments}

The specimens of the hardness test were divided randomly into four equal groups $(n=15)$. The specimens of the tensile test were divided randomly into five equal groups $(n=12)$. One group served as control in the tensile test and treated with distilled water. In the SDF group, the specimens underwent a topical application of a 30\% SDF solution (Ancarie, Cariostatico, Maquira Dental Products, Maringa, PR, Brazil) for 3 minutes. ${ }^{26}$ In the GSE group, the specimens were immersed in $6.5 \%$ 
GSE solution for 10 minutes, which was prepared using $6.5 \mathrm{gr}$ of GSE in the form of powder (Puritans Pride Inc, Oakdale, New York, United States), dissolved in $100 \mathrm{~mL}$ of distilled water. ${ }^{27} \mathrm{In}$ the GSE+SDF group, first the specimens were immersed in GSE solution and then SDF solution was applied. In the SDF+GSE group, first SDF solution was applied and then the specimens were immersed in GSE solution. The specimens were kept under $100 \%$ humidity at $37^{\circ} \mathrm{C}$ before the mechanical test.

\section{Mechanical Tests}

Microhardness measurements were taken before initial exposure to the experimental solutions (baseline) and after treatment. The specimens were positioned perpendicular to the long axis of indenter in microhardness testing machine (SCTMC, MHV-1000Z, PRC) to record the Knoop hardness number (KHN). The test load was increased at a constant rate to $25 \mathrm{~kg} \mathrm{~F} / \mathrm{mm}^{2}$ in 20 seconds. Three measurements were made for each sample and the mean value was established as the KHN.

In order to measure elastic modulus, the trimmed specimens were glued to a tensile testing jig by means of cyanoacrylate adhesive (Zapit, Dental Ventures of America, Corona, California, United States). The jig was pulled at a rate of $0.6 \mathrm{~mm} / \mathrm{min}$ in a universal testing machine (Instron; Zwich, Germany) and modulus of elasticity was recorded in MPa.

\section{Statistical Analysis}

The baseline hardness values were analyzed with one-way analysis of variance (ANOVA) to investigate the normal distribution of the specimens among the groups. $\mathrm{H}_{1}-\mathrm{H}_{0}$ was defined by subtracting baseline hardness from the final hardness. Normality of $\mathrm{H}_{1}-\mathrm{H}_{0}$ values was evaluated by Kolmogorov-Smirnov test and one-way ANOVA revealed significant difference between the groups. The difference in $\mathrm{H}_{1}-\mathrm{H}_{0}$ values among the groups were analyzed with Tamhane multiple comparisons. Paired sample $t$-test was used to analyze the difference between the baseline and final hardness of each group. Data for modus of elasticity were analyzed with one-way ANOVA. The differences between the treatments were identified and compared with Tamhane multiple comparison tests. All the comparisons were made at a significance level of 0.05 .

\section{Results}

The results of the mechanical tests are summarized in - Table 1. One-way ANOVA showed no significant differences in the baseline hardness between the study groups ( $p=0.07$ ). One-way ANOVA revealed significant differences in $\mathrm{H}_{1}-\mathrm{H}_{0}$ values between the study groups $(p=0.00)$. Significant differences were seen when the $\mathrm{H}_{1}-\mathrm{H}_{0}$ values of the groups were compared using Tamhane multiple comparisons (SDF>SD$\mathrm{F}+\mathrm{GSE}>\mathrm{GSE}>\mathrm{GSE}+\mathrm{SDF}$ ). Paired sample $t$-test revealed $\mathrm{a}$ significant difference between the baseline and final hardness in each group $(p=0.00)$. One-way ANOVA revealed statistically significant differences in elastic modulus between the groups $(p=0.00)$. Elastic modulus of caries-affected dentin (control) was significantly lower than the SDF and SDF+GSE groups. $p$-values were 0.00 and 0.02 , respectively, but when compared to other groups, no significant differences were detected.

\section{Discussion}

The results of the present study led to the rejection of the null hypothesis. SDF and SDF+GSE solutions were capable of increasing hardness and elastic modulus of inner carious dentin. Although GSE and GSE+SDF solutions increased hardness of inner carious dentin, they could not significantly increase elastic modulus. Hardness and elastic modulus have been indicated to be related to the mineral content of calcified tissues. ${ }^{28,29}$ The reduction in the mechanical properties of carious dentin is due to the loss of minerals. Weight percentage of mineral content was found to determine hardness and elastic modulus of carious primary dentin exponentially. ${ }^{29}$ However, porosity that increases with mineral loss is also a determining factor of elastic modulus. ${ }^{30}$ Some studies proposed that in the absence of intrafibrillar minerals the mineral content and mechanical properties are not linearly correlated. These studies suggested that a reliable indicator of functional remineralization of affected dentin is re-establishment of mechanical properties. ${ }^{9,11}$ Remineralization of dentin can occur either by precipitation of minerals between collagen fibrils in an extrafibrillar manner or by functional bonding of minerals to the fibrils in an intrafibrillar manner. ${ }^{11}$ When the gap zones of collagen fibrils are filled by intrafibrillar remineralization, porosity of carious dentin might be reduced.

SDF treatment resulted in the greatest improvement in mechanical properties of carious dentin compared to other groups. This can be related to increased mineral content. Surface mineralization (increased hardness) and functional mineralization (increased elastic modulus) were evident in

Table 1 Means (standard deviation) of Knoop hardness and modulus of elasticity

\begin{tabular}{|l|l|l|l|l|}
\hline Study groups & $\mathbf{H}_{0}$ & $\mathbf{H}_{1}$ & $\mathbf{H}_{1}-\mathbf{H}_{0}$ & $\mathbf{E}(\mathrm{MPa})$ \\
\hline Control & - & - & - & $2.11(0.97)^{\mathrm{A}}$ \\
\hline SDF & $8.31(0.67)^{\mathrm{A}, \mathrm{a}}$ & $21.91(3.78)^{\mathrm{b}}$ & $13.60(3.83)^{\mathrm{A}}$ & $5.67(0.88)^{\mathrm{B}, \mathrm{C}}$ \\
\hline GSE & $9.53(1.34)^{\mathrm{A}, \mathrm{a}}$ & $14.72(2.73)^{\mathrm{b}}$ & $5.01(1.98)^{\mathrm{B}}$ & $3.90(1.39)^{\mathrm{A}, \mathrm{C}, \mathrm{E}}$ \\
\hline GSE+SDF & $8.59(1.52)^{\mathrm{A}, \mathrm{a}}$ & $11.05(1.63)^{\mathrm{b}}$ & $2.46(0.87)^{\mathrm{C}}$ & $2.51(0.64)^{\mathrm{A}, \mathrm{D}, \mathrm{E}}$ \\
\hline SDF+GSE & $8.92(1.42)^{\mathrm{A}, \mathrm{a}}$ & $17.96(1.69)^{\mathrm{b}}$ & $9.04(2.20)^{\mathrm{D}}$ & $4.79(1.95)^{\mathrm{B}, \mathrm{C}, \mathrm{E}}$ \\
\hline
\end{tabular}

Abbreviations: GSE, grape seed extract; SDF, silver diamine fluoride.

${ }^{*} \mathrm{H}_{0}$, baseline hardness; $\mathrm{H}_{1}$, post-treatment hardness; $\mathrm{E}(\mathrm{MPa})$, elastic modulus (mega pascal).

** Same upper-case letters indicate no significant difference within each column. Same lower-case letters indicate no significant difference within each column. 
the SDF group. This was consistent with previous studies. ${ }^{21,22}$ Mei et al observed a dense granular structure of spherical grains under SEM on the surface of the demineralized dentin treated with SDF. This might be an indicator for extrafibrillar mineral formation. Their earlier study found that the microhardness of remineralized dentin increased after SDF treatment. ${ }^{31,32}$ They reported that remineralization of demineralized dentin with SDF causes extrafibrillar and intrafibrillar mineral formation. ${ }^{21}$ Available evidence indicates that elasticity and stiffness of demineralized collagen tissue are strongly dependent on the intrafibrillar mineral formation. ${ }^{9,12}$

In the present study, elastic modulus of GSE-treated carious dentin increased but it was not significantly different from the control group. During caries process, collagen crosslinks break down and expose organic matrix, leaving behind demineralized collagen matrix prone to degradation. Therefore, preserving collagen scaffold is mandatory for repair of the carious lesion. ${ }^{33}$ GSE as a natural agent can interact with dentin collagen, improve its mechanical properties, ${ }^{15,34}$ and decrease the degradation rates of sound and caries-affected dentine. ${ }^{35}$ It is also an MMP's inhibitor and reduces collagen degradation..$^{34,36}$ The elastic modulus of GSE treated demineralized dentin matrices showed improvements. ${ }^{34}$ Previous studies used completely demineralized dentin matrices. However, the inner carious dentin specimens used in this study are specified with decreased minerals and collagen cross-links but without protein denaturation. ${ }^{37}$ Remaining mineral crystals that form electrostatic bonds with collagen fibrils and the distance between the fibers, ${ }^{38}$ owing to the presence of extraand intrafibrillar minerals, might restrict formation of more cross-links. As a result, longer periods of GSE treatment may become necessary in order to observe any significant changes in collagen's mechanical properties. It has been shown that cross-linking degree of demineralized dentin collagen treated with cross-linking agents is time-dependent. ${ }^{39}$ There is no consensus on the interaction mechanism of PA with collagen. Four different mechanisms are proposed: covalent interaction, ionic interaction, hydrogen bonding interaction, or hydrophobic interactions. ${ }^{40}$ One of the accepted mechanisms is the formation of calcium ion bridges between the telopeptide ends of matrix collagen and hydroxyapatite that dissolves during demineralization. ${ }^{41}$ These calcium links are supposed to deal with collagen elasticity. ${ }^{41}$ Hydrogen bonding between amine carboxyl and the phenolic hydroxyl groups is the other plausible mechanism of interaction between PA and collagen. ${ }^{42}$ Hardness increased after GSE treatment. Established cross-links might increase the resistance of surface dentin to deformation by the load applied through the indenter of microhardness tester. GSE formed insoluble complexes in water. These complexes remained visually insoluble at natural $\mathrm{pH}^{4}{ }^{43}$ These complexes might contribute to the increased hardness. Pavan et al also observed a wellmineralized layer on the surface of GSE-treated carious dentin specimens. ${ }^{44}$ However, they used a remineralizing $\mathrm{pH}$ cycling model after the experimental treatments.

GSE+SDF treatment increased hardness of inner carious dentin but not the elastic modulus. However, hardness of this group was the lowest among other treated groups. This can be attributed to the chemical interaction of GSE with fluoride. ${ }^{44}$ GSE present on the surface of the specimens scavenges fluoride ions of SDF and prevents the complete interaction of SDF with hydroxyapatite. Another explanation can be the "fast" process of remineralization in which cross-linked collagen matrix prevents ion-diffusion into the deeper parts of the lesion. ${ }^{45}$ Cross-linked collagen might have limited or no sites for mineral bonding, inhibiting intra-fibrillar mineral formation. As mentioned earlier, in contrast to hardness, elastic modulus is correlated with porosity. Hiraishi et $\mathrm{al}^{46}$ and Xie et $\mathrm{al}^{43}$ found that hesperidin and GSE promoted dentin remineralization. This discrepancy can be attributed to the different mechanisms used in these studies for remineralization. They used a $\mathrm{pH}$ cycling method that allowed for gradual mineral diffusion into the lesion.

Hardness of caries-affected dentin in the SDF+GSE group could not achieve the values in the SDF group, but the elastic modulus of these two groups was not different. Mei et $\mathrm{al}^{21}$ proposed the hypothesis that SDF could interact with exposed collagen. This might reduce the affinity of collagen for interaction with GSE.

The results of the present study did not support any synergistic effect between SDF and GSE. It seems that SDF alone remineralizes and strengthens caries-affected dentin in only one step and shorter clinical application time. GSE adds no benefits to this agent because SDF deals with both the mineral content and collagen network. It can interact with collagen fibers and protect them from denaturation. Also, it can inactivate endogenous proteases that become active by the low $\mathrm{pH}$ of carious challenge.

Previous studies ${ }^{21,33,43}$ aimed to investigate the effect of SDF or GSE on demineralized dentin, pH-cycled the specimens treated with the two agents, and then evaluated the remineralization outcome. They did not consider the effect of SDF and GSE solutions alone. This study was the first to measure the effect of SDF and GSE on mechanical properties of carious dentin and a parallel study aims to investigate the effect of these solutions on demineralization resistance and remineralization capacity of carious dentin.

Specimen size and shape and also unavoidable differences in tubular structure and orientation would affect mechanical test results. Moreover, carious lesions are not similar, ${ }^{47}$ that is, carious process adds to this heterogeneity. Attempts to simulate carious lesion in vitro were not very successful. Caries-affected dentin created in vitro by demineralizing normal dentin is completely demineralized with empty tubules but natural caries-affected dentin is the result of several cycles of demineralization and remineralization. The tubules are partially obstructed with whitlockite crystals. ${ }^{4}$ As with many other studies, ${ }^{4,10}$ natural carious teeth were used in the present study. Teeth with similar caries depth were selected by means of radiography. Caries-detector solution, visual inspection, and a standard scale (North Carolina Dentin Sclerosis Scale) were used to manage the variations of the carious lesions. Nondestructive tests are the best way to study natural carious lesions (e.g., hardness test, nanoindentation test).

It was not possible to measure the Knoop hardness of wet specimens but modulus of elasticity was measured in wet state, which is more clinically relevant. 


\section{Conclusion}

Within the limitations of the current study, SDF solution can be recommended to increase hardness and elastic modulus of caries-affected dentin. GSE treatment after SDF application was shown to improve mechanical properties of caries-affected dentin but no extra benefit was achieved compared to SDF alone. Although GSE and GSE+SDF increased hardness, they could not affect elastic modulus of cariesaffected dentin.

This article has not been published and is not under consideration for publication elsewhere. We have no conflicts of interest to disclose. This article has been read and approved by all the authors, the requirements for authorship have been met, and each author believes that the manuscript represents honest work.

\section{Funding}

This research (Grant\#8793084) was carried out by the financial support from the Deputy Dean of Research at Shiraz University of Medical Sciences.

\section{Conflict of Interest}

None declared.

\section{Acknowledgements}

The authors thank Dr. Mahrdad Vosoughi for statistical analysis of data and Dr. Majid Abdolrahimi for revising the English manuscript.

\section{References}

1 Featherstone JD. Modeling the caries-inhibitory effects of dental materials. Dent Mater 1996;12(3):194-197

2 Chaussain-Miller C, Fioretti F, Goldberg M. Menashi S. The role of matrix metalloproteinases (MMPs) in human caries. J Dent Res 2006;85(1):22-32

3 Tersariol IL, Geraldeli S, Minciotti CL, et al. Cysteine cathepsins in human dentin-pulp complex. J Endod 2010;36(3):475-481

4 Ito S, Saito T, Tay FR, Carvalho RM, Yoshiyama M, Pashley DH. Water content and apparent stiffness of non-caries versus caries-affected human dentin. J Biomed Mater Res B Appl Biomater 2005;72(1):109-116

5 Kuboki Y, Ohgushi K, Fusayama T. Collagen biochemistry of the two layers of carious dentin.J Dent Res 1977;56(10):1233-1237

6 Zheng L, Hilton JF, Habelitz S, Marshall SJ, Marshall GW. Dentin caries activity status related to hardness and elasticity. Eur J Oral Sci 2003;111(3):243-252

7 Pugach MK, Strother J, Darling CL, et al. Dentin caries zones: mineral, structure, and properties. J Dent Res 2009;88(1):71-76

8 Macedo de Lima JF, Wajngarten D, Islam F, Clifford J, Botta AC. Effect of adhesive mode and chlorhexidine on microtensile strength of universal bonding agent to sound and cariesaffected dentins. Eur J Dent 2018;12(4):553-558

9 Kinney JH, Marshall SJ, Marshall GW. The mechanical properties of human dentin: a critical review and re-evaluation of the dental literature. Crit Rev Oral Biol Med 2003;14(1):13-29

10 Nishitani Y, Yoshiyama M, Tay FR, et al. Tensile strength of mineralized/demineralized human normal and carious dentin. J Dent Res 2005;84(11):1075-1078

11 Bertassoni LE, Habelitz S, Kinney JH, Marshall SJ, Marshall GW, Jr. Biomechanical perspective on the remineralization of dentin. Caries Res 2009;43(1):70-77
12 Balooch M, Habelitz S, Kinney JH, Marshall SJ, Marshall GW. Mechanical properties of mineralized collagen fibrils as influenced by demineralization. J Struct Biol 2008;162(3):404-410

13 Hara AT, Ando M, Cury JA, Serra MC, González-Cabezas C, Zero DT. Influence of the organic matrix on root dentine erosion by citric acid. Caries Res 2005;39(2):134-138

$14 \mathrm{Xu} \mathrm{C}$, Wang Y. Cross-linked demineralized dentin maintains its mechanical stability when challenged by bacterial collagenase. J Biomed Mater Res B Appl Biomater 2011;96(2):242-248

15 Bedran-Russo AK, Pereira PN, Duarte WR. Drummond JL, Yamauchi M. Application of crosslinkers to dentin collagen enhances the ultimate tensile strength. J Biomed Mater Res B Appl Biomater 2007;80(1):268-272

16 Carrilho MR, Tay FR, Donnelly AM, et al. Host-derived loss of dentin matrix stiffness associated with solubilization of collagen. J Biomed Mater Res B Appl Biomater 2009;90(1):373-380

17 Hiraishi N, Sono R, Sofiqul I, et al. In vitro evaluation of plant-derived agents to preserve dentin collagen. Dent Mater 2013;29(10):1048-1054

18 Liu Y, Chen M, Yao X, Xu C, Zhang Y, Wang Y. Enhancement in dentin collagen's biological stability after proanthocyanidins treatment in clinically relevant time periods. Dent Mater 2013;29(4):485-492

19 Knight GM, McIntyre JM, Craig GG, Mulyani; , Zilm PS, Gully NJ. Differences between normal and demineralized dentine pretreated with silver fluoride and potassium iodide after an in vitro challenge by Streptococcus mutans. Aust Dent J 2007;52(1):16-21

20 Mei ML, Chu $\mathrm{CH}$, Low KH, Che CM, Lo EC. Caries arresting effect of silver diamine fluoride on dentine carious lesion with S. mutans and L. acidophilus dual-species cariogenic biofilm. Med Oral Patol Oral Cir Bucal 2013;18(6):e824-e831

21 Mei ML, Ito L, Cao Y, Li QL, Lo EC, Chu CH. Inhibitory effect of silver diamine fluoride on dentine demineralisation and collagen degradation. J Dent 2013;41(9):809-817

22 Mei ML, Li QL, Chu CH, Yiu CK, Lo EC. The inhibitory effects of silver diamine fluoride at different concentrations on matrix metalloproteinases. Dent Mater 2012;28(8):903-908

23 Hevinga MA, Opdam NJ, Frencken JE, Truin GJ, Huysmans MC. Does incomplete caries removal reduce strength of restored teeth? J Dent Res 2010;89(11):1270-1275

24 Movahhedian N, Adibi S, Tavakoli HS, Baseri H. How does triangular-shaped radiolucency affect caries diagnosis? Oral Radiol 2017;33(1):32-37

25 Doozandeh M, Firouzmandi M, Mirmohammadi M. The simultaneous effect of extended etching time and casein phosphopeptide-amorphous calcium phosphate containing paste application on shear bond strength of etch-and-rinse adhesive to caries-affected dentin. J Contemp Dent Pract 2015;16(10):794-799

26 Quock RL, Barros JA, Yang SW, Patel SA. Effect of silver diamine fluoride on microtensile bond strength to dentin. Oper Dent 2012;37(6):610-616

27 Shafiei F, Firouzmandi M, Zamanpour M. The effect of two cross-linking agents on dentin bond strength of resinmodified glass ionomer. Eur J Dent 2017;11(4):486-490

28 Angker L, Swain MV, Kilpatrick N. Characterising the micromechanical behaviour of the carious dentine of primary teeth using nano-indentation. J Biomech 2005;38(7):1535-1542

29 Angker L, Nockolds C, Swain MV, Kilpatrick N. Correlating the mechanical properties to the mineral content of carious dentine--a comparative study using an ultra-micro indentation system (UMIS) and SEM-BSE signals. Arch Oral Biol 2004;49(5):369-378

30 Currey JD. The effect of porosity and mineral content on the Young's modulus of elasticity of compact bone. J Biomech 1988;21(2):131-139 
$31 \mathrm{Chu} \mathrm{CH}$, Lo EC. Microhardness of dentine in primary teeth after topical fluoride applications. J Dent 2008;36(6):387-391

$32 \mathrm{Chu}$ CH, Mei L, Seneviratne CJ, Lo EC. Effects of silver diamine fluoride on dentine carious lesions induced by Streptococcus mutans and Actinomyces naeslundii biofilms. Int J Paediatr Dent 2012;22(1):2-10

33 Epasinghe DJ, Kwan S, Chu D, Lei MM, Burrow MF, Yiu CKY. Synergistic effects of proanthocyanidin, tri-calcium phosphate and fluoride on artificial root caries and dentine collagen. Mater Sci Eng C 2017;73:293-299

34 Bedran-Russo AK, Pashley DH, Agee K. Drummond JL, Miescke $\mathrm{KJ}$. Changes in stiffness of demineralized dentin following application of collagen crosslinkers. J Biomed Mater Res B Appl Biomater 2008;86(2):330-334

35 Macedo GV, Yamauchi M, Bedran-Russo AK. Effects of chemical cross-linkers on caries-affected dentin bonding. J Dent Res 2009;88(12):1096-1100

36 Epasinghe DJ, Yiu CK, Burrow MF, Hiraishi N, Tay FR. The inhibitory effect of proanthocyanidin on soluble and collagen-bound proteases. J Dent 2013;41(9):832-839

37 Nakornchai S, Atsawasuwan P, Kitamura E, Surarit R, Yamauchi M. Partial biochemical characterisation of collagen in carious dentin of human primary teeth. Arch Oral Biol 2004;49(4):267-273

38 Koide T, Daito M. Effects of various collagen crosslinking techniques on mechanical properties of collagen film. Dent Mater J 1997;16(1):1-9
39 Liu R, Fang M, Xiao Y, et al. The effect of transient proanthocyanidins preconditioning on the cross-linking and mechanical properties of demineralized dentin. J Mater Sci Mater Med 2011;22(11):2403-2411

40 Al-Ammar A, Drummond JL, Bedran-Russo AK. The use of collagen cross-linking agents to enhance dentin bond strength. J Biomed Mater Res B Appl Biomater 2009;91(1):419-424

41 Hellmich Ch, Ulm FJ. Are mineralized tissues open crystal foams reinforced by crosslinked collagen? Some energy arguments. J Biomech 2002;35(9):1199-1212

42 Han B, Jaurequi J, Tang BW, Nimni ME. Proanthocyanidin: a natural crosslinking reagent for stabilizing collagen matrices. J Biomed Mater Res A 2003;65(1):118-124

43 Xie $\mathrm{Q}$, Bedran-Russo AK, Wu CD. In vitro remineralization effects of grape seed extract on artificial root caries. J Dent 2008;36(11):900-906

44 Pavan S, Xie Q Hara AT, Bedran-Russo AK. Biomimetic approach for root caries prevention using a proanthocyanidin-rich agent. Caries Res 2011;45(5):443-447

45 ten Cate JM. Remineralization of caries lesions extending into dentin. J Dent Res 2001;80(5):1407-1411

46 Hiraishi N, Sono R, Islam MS, Otsuki M, Tagami J, Takatsuka T. Effect of hesperidin in vitro on root dentine collagen and demineralization. J Dent 2011;39(5):391-396

47 Marshall GW, Habelitz S, Gallagher R, Balooch M, Balooch G, Marshall SJ. Nanomechanical properties of hydrated carious human dentin. J Dent Res 2001;80(8):1768-1771 